\title{
Sequential Organ Failure Assessment score as a predictor of mortality in ventilated patients with multidrug-resistant bacteremia
}

\author{
Yeseul Oh, Jiyeon Roh, Jaemin Lee, Hyun Sung Chung, Kwangha Lee, Min Ki Lee \\ Division of Pulmonary, Allergy, and Critical Care Medicine, Department of Internal Medicine, Pusan National University School of Medicine, Busan, Korea
}

Background: The occurrence of multidrug-resistant (MDR) bacteremia in ventilated patients may be associated with a high mortality rate. We evaluated whether Sequential Organ Failure Assessment (SOFA) score on the day of bacteremia could predict 90-day mortality in these patients.

Methods: Data were obtained retrospectively from 202 patients (male, 60.4\%; median age, 64 years) hospitalized at a single university-affiliated tertiary care hospital. All adult patients who had were ventilated and had one of the following six MDR bacteremias between March 2011 and February 2018 were enrolled: methicillin-resistant Staphylococcus aureus, extended-spectrum $\beta$-lactamase-producing Gram-negative bacteria (Escherichia coli and Klebsiella pneumonia), carbapenem-resistant Gram-negative rods (Acinetobacter baumannii and Pseudomonas aeruginosa), or vancomycin-resistant Enterococcus faecium.

Results: The overall 90-day mortality rate after the day of bacteremia was 59.9\%. The areas under the receiver operating characteristic curves for the SOFA and Acute Physiology and Chronic Health Evaluation (APACHE) II scores were 0.732 (95\% confidence interval [CI], 0.666 to $0.792 ; \mathrm{P}<0.001)$ and $0.662(95 \% \mathrm{Cl}, 0.593$ to $0.727 ; \mathrm{P}<0.001)$, respectively, with no difference between the two $(P=0.059)$. Also, the cutoff value of the SOFA score was 9 (based on Youden's index). Multivariate Cox regression analysis showed that this cut-off value was significantly associated with higher mortality rate (hazard ratio, 2.886; $95 \% \mathrm{Cl}, 1.946$ to 4.221 ; $\mathrm{P}<0.001)$.

Conclusions: SOFA score measured on the day of bacteremia may be a useful prognostic indicator of 90-day mortality in ventilated patients with MDR bacteremia.

Key Words: bacteremia; intensive care unit; prognosis

\section{INTRODUCTION}

Although clinical guidelines have recommended reductions in empirical treatment with broad-spectrum antibiotics, timely administration of more appropriate antibiotics, and development of strict infection control policies [1-5], the incidence of serious complications caused by multidrug-resistant (MDR) bacteria has been increasing in intensive care unit (ICU) patients [6]. Of these complications, the presence of MDR bacteria in the bloodstream (bacteremia) can have serious health consequences, including sepsis, septic shock, and he-

\section{Original Article}

Received: March 20, 2020

Revised: June 10, 2020

Accepted: June 25, 2020

Corresponding author

Kwangha Lee

Division of Pulmonary, Allergy, and

Critical Care Medicine, Department

of Internal Medicine, Pusan National

University School of Medicine, 179

Gudeok-ro, Seo-gu, Busan 49241,

Korea

Tel: $+82-51-240-7743$

Fax: +82-51-245-3127

E-mail: jubilate@pusan.ac.kr

Copyright $\odot 2020$ The Korean Society of Critical Care Medicine

This is an Open Access article distributed under the terms of Creative Attributions Non-Commercial License (https:// creativecommons.org/li-censes/by-nc/4.0/) which permits unrestricted noncommercial use, distribution, and reproduction in any medium, provided the original work is properly cited. 
matogenous spread to other organs. These sequelae are associated with increased need for medical resources over long periods of time and high medical costs.

Notably, occurrence of MDR bacteremia in ventilated ICU patients may result in longer ICU and hospital length of stay and higher mortality rates despite intensive resource utilization, including treatment with antibiotics. Therefore, identification of clinical factors associated with poor prognosis, including mortality, may allow future health care planning. Moreover, critical care resources and facilities, even at university hospitals, are frequently limited in developing countries [7-9]. Few studies to date, however, have assessed prognostic indicators in patients with MDR bacteremia who received mechanical ventilation.

Because bacteremia is frequently accompanied by sepsis, and sepsis is diagnosed based on Sequential Organ Failure Assessment (SOFA) score, the present study hypothesized that SOFA score on the first day of bacteremia would be useful for predicting patient prognosis $[10,11]$. Therefore, the aim of the present study was to investigate whether SOFA score would be useful for predicting mortality after the day of blood culture in ventilated patients with MDR bacteremia. In addition, the prognostic ability of the SOFA score was compared with that of the Acute Physiology and Chronic Health Evaluation (APACHE) II score on the day of bacteremia [12].

\section{MATERIALS AND METHODS}

\section{Study Design and Subjects}

This retrospective study included patients admitted to the ICUs of a 1,100-bed university-affiliated tertiary care hospital from March 1, 2011 to February 28, 2018. This hospital has six functionally separate ICUs with a total of 85 beds, including 12 in the medical, 10 in the surgical, 14 in the cardio-stroke, 13 in the neurosurgical, 20 in the emergency, and 16 in the trauma ICU. All of these ICUs have full cardiovascular and close airway monitoring equipment, as well as one full-time ICU specialist; the nurse-to-bed ratio is 1:3. All subjects were managed according to therapeutic recommendations, which were based on a lung-protective ventilator strategy [13].

Records of all adult patients aged $\geq 18$ years admitted to these six ICUs were screened to determine whether they had received ventilator care and had a positive blood culture during the study period. Patients were finally enrolled if they had one of the following six MDR bacteremias: methicillin-resistant Staphylococcus aureus, extended-spectrum $\beta$-lactamaseproducing Gram-negative bacteria (Escherichia coli and Kleb-

\section{KEY MESSAGES}

- Occurrence of multidrug-resistant (MDR) bacteremia in ventilated intensive care unit patients may result in longer hospital length of stay and higher mortality rates.

- Sequential Organ Failure Assessment score measured on the day of bacteremia may be a useful prognostic indicator of 90-day mortality in ventilated patients with MDR bacteremia.

siella pneumoniae), carbapenem-resistant Gram-negative rods (Acinetobacter baumannii and Pseudomonas aeruginosa), or vancomycin-resistant Enterococcus faecium. Patients with polymicrobial bacteremia and those who experienced a subsequent episode of bacteremia within 3 days after the first (index) episode of bacteremia were excluded [14]. Organisms in all positive blood cultures were identified by matrix-assisted laser desorption/ionization time-of-flight mass spectrometry (MALDI-TOF MS; Bruker Daltonics, Bremen, Germany). Antimicrobial susceptibility was assessed using the VITEK-2 and E-tests (bioMérieux, Marcy l'Etoile, France). The primary outcome was all-cause mortality 90 days after bacteremia.

All investigators confirmed that the study objectives and procedures were disclosed honestly to all patients, and all investigators had full access to all data. Case report forms, including all relevant medical, laboratory, and radiological data, were completed for each included patient. The study protocol was approved by the Institutional Review Board of Pusan National University Hospital (IRB No. 1904-004-077), which waived the requirement for informed consent from enrolled patients or their surrogates because of the observational nature of this study. This study had no impact on the future treatment of enrolled patients.

\section{Data Collection}

Demographic and clinical data were obtained retrospectively from the electronic medical records of each subject; these included age, sex, comorbidities before ICU admission, duration of ventilator care, and length of stay in the ICU and hospital. Severity of illness was measured using the APACHE II score, and accompanying organ failure was assessed according to the SOFA score [10,12], with both scores calculated from laboratory and clinical data recorded on the day of blood culture. Diagnosis of acute respiratory distress syndrome (ARDS) on the day of bacteremia was based on the Berlin Definition [15].

The recorded clinical data included the primary sources of infection and requirements for hemodialysis (defined as any 


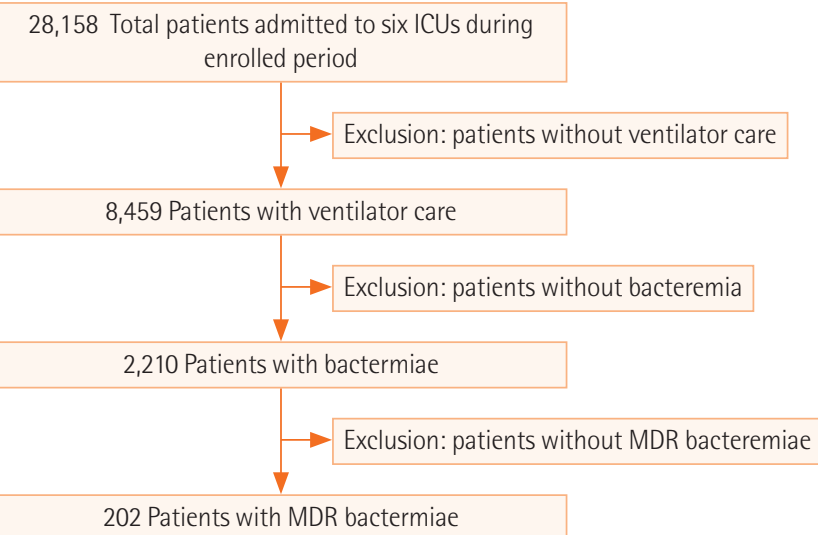

Figure 1. Flowchart of recruited and enrolled study participants. ICU: intensive care unit; MDR: multidrug-resistant.

form of renal replacement therapy), neuromuscular blocking agents, and vasopressors on the day of blood culture. Because no standardized nationwide antimicrobial stewardship program addressing increased antimicrobial resistance has been recognized internationally [16], this study also assessed whether the choice of antimicrobial agents was based on consultations with a specialist in infectious diseases.

In-hospital mortality was defined as death prior to hospital discharge, whereas 90-day mortality was defined as mortality within 90 days of the day of blood culture. Survival status through February 28, 2019 was assessed in all patients by review of the National Health Insurance Service Database.

\section{Statistical Analysis}

Continuous variables are expressed as the median (range) and were compared using Student t-test or the Mann-Whitney U-test, as applicable. Categorical variables are expressed as numbers (percentages) and were compared using the chisquare test or Fisher's exact test (for small numbers), as appropriate. Receiver operating characteristics (ROC) curves and the areas under the ROC curves (AUCs) for SOFA scores were calculated, and an optimal cut-off value for the SOFA score was determined based on the maximum Youden's index [17]. The sensitivity (SS), specificity (SP), positive likelihood ratio (PLR), negative likelihood ratio (NLR), positive predictive value (PPV), and negative predictive value (NPV) of SOFA scores for predicting mortality were determined. Univariate Cox proportional hazard models were developed to determine the relationships between SOFA scores and other clinical variables with mortality. Variables with $\mathrm{P}<0.1$ on univariate models were included in multivariate Cox proportional hazard models, followed by backward elimination of any fac-
Table 1. Demographic and clinical characteristics of all enrolled patients

\begin{tabular}{|c|c|}
\hline Characteristics & Total $(n=202)$ \\
\hline Age (yr) & $64(18-95)$ \\
\hline Male sex & $122(60.4)$ \\
\hline Hospital LOS (day) & $40(2-431)$ \\
\hline ICU LOS (day) & $23(1-430)$ \\
\hline Duration of MV (day) & $17(1-430)$ \\
\hline \multicolumn{2}{|l|}{ Comorbidity } \\
\hline Cardiovascular disease & $63(31.2)$ \\
\hline Diabetes mellitus & $55(27.2)$ \\
\hline Hemato-oncological diseases & $43(21.3)$ \\
\hline Cerebrovascular diseases & $31(15.3)$ \\
\hline Chronic renal diseases & $27(13.4)$ \\
\hline Chronic lung diseases & $15(7.4)$ \\
\hline Chronic liver diseases & $13(6.4)$ \\
\hline Neuromuscular diseases & $12(5.9)$ \\
\hline Biliary diseases & $9(4.5)$ \\
\hline Alimentary diseases & $8(4.0)$ \\
\hline \multicolumn{2}{|l|}{ Main infection source } \\
\hline Pneumonia & $98(48.5)$ \\
\hline Vascular catheter-related & $46(22.8)$ \\
\hline Intra-abdominal & $25(12.4)$ \\
\hline Musculoskeletal and soft tissue & $15(7.4)$ \\
\hline Urinary & $9(4.5)$ \\
\hline Other ${ }^{\mathrm{a}}$ & $9(4.5)$ \\
\hline Period from ICU admission to bacteremia (day) & $9(0-360)$ \\
\hline APACHE II score ${ }^{b}$ & $19(5-38)$ \\
\hline SOFA score ${ }^{b}$ & $9(2-20)$ \\
\hline \multicolumn{2}{|l|}{ Microorganism identified } \\
\hline Methicillin-resistant Staphylococcus aureus & $77(38.1)$ \\
\hline Vancomycin-resistant Enterococcus faecium & $25(12.4)$ \\
\hline Carbapenem-resistant Acinetobacter baumannii & $63(31.2)$ \\
\hline ESBL(+) Klebsiella pneumoniae & $25(12.4)$ \\
\hline ESBL(+) Escherichia coli & $18(8.9)$ \\
\hline Carbapenem-resistant Pseudomonas aeruginosa & $5(2.5)$ \\
\hline Requirement for vasopressors ${ }^{b}$ & $113(55.9)$ \\
\hline Requirement for hemodialysis ${ }^{b}$ & $56(29.2)$ \\
\hline Requirement for neuromuscular blocking agents ${ }^{b}$ & $51(25.2)$ \\
\hline ARDS on the day of bacteremia & $153(75.7)$ \\
\hline $\begin{array}{l}\text { ID consultations by ID specialists regarding } \\
\text { management of bacteremia }\end{array}$ & $128(63.4)$ \\
\hline Period from day of bacteremia to hospital discharge (day) & $18(0-373)$ \\
\hline In-hospital mortality & $115(56.9)$ \\
\hline 90-Day mortality after bacteremia & $121(59.9)$ \\
\hline
\end{tabular}

Values are presented as median (range) or number (\%).

LOS: length of stay; ICU: intensive care unit; MV: mechanical ventilation; APACHE: Acute Physiology and Chronic Health Evaluation; SOFA: Sequential Organ Failure Assessment; ESBL: extended-spectrum $\beta$-lactamase-producing; ARDS: acute respiratory distress syndrome; ID: infectious diseases.

${ }^{a}$ Other included meningitis $(n=3)$, infective endocarditis $(n=3)$, ventriculo-peritoneal shunt infection $(n=2)$, and retropharyngeal abscess $(n=1)$; ${ }^{b}$ All clinical data were calculated or obtained from medical records on the day of blood collection. 
tor with a P-value $>0.05$ in the multivariate model. Hazard ratios (HRs) and 95\% confidence intervals (CIs) were calculated. Kaplan-Meier estimates of mortality were stratified according to the SOFA score cutoff values developed in this study, and curves (total and subgroups) were compared using log-rank tests. The AUCs of SOFA scores and APACHE II scores for 90day mortality were compared using DeLong's test [18]. All tests were two-tailed, and P-values $<0.05$ were considered statistically significant. All analyses were performed using IBM SPSS ver. 24.0 (IBM Corp., Armonk, NY, USA) and MedCalc version 18.11.6 (MedCalc, Ostend, Belgium).

\section{RESULTS}

\section{General Characteristics}

During the study period, 28,158 patients were admitted to one of the six ICUs. Of these, 202 patients (1.1\%) were eligible for participation in the study (Figure 1). Table 1 shows the baseline characteristics and clinical outcomes of these patients. The median period from ICU admission to bacteremia was 9 days (range, 0-360 days). Pneumonia was the most common source of bacteremia $(n=98,48.5 \%)$. When we further evaluated these patients to see whether the same organisms would be identified in tracheal aspirates and bronchoscopic alveolar lavage specimens, 83 patients ( $84.7 \%$ ) had the same organism in these respiratory specimens. Of the 202 patients, 100 (49.5\%) and 106 (52.5\%) patients had Gram-positive and Gram-negative bacteremia, respectively, with four $(2.0 \%)$ patients having both. In addition, 153 patients (75.7\%) were diagnosed with ARDS on the day of bacteremia, and 128 patients (63.4\%) consulted with an infectious diseases specialist for positive blood culture results. The in-hospital and 90-day mortality rates were $56.9 \%$ and $59.9 \%$, respectively.

\section{Predicting Mortality Using SOFA Score}

ROC curve analyses were performed to determine the SOFA score cutoffs for predicting mortality, as well as the SS, SP, PLR, NLR, PPV, and NPV of each, in all patients and in those with Gram-positive or Gram-negative bacteremia. In total patients, the AUC was 0.732 (95\% CI, 0.666 to $0.792 ; \mathrm{P}<0.001$; SS, $72.8 \%$; SP, 65.3\%; PLR, 2.1; NLR, 0.4; PPV, 58.4\%; NPV, 78.2\%) and the cutoff value was 9. The AUC of patients with Gram-positive bacteremia was 0.733 (95\% CI, 0.666 to $0.792 ; \mathrm{P}<0.001$; SS, $67.4 \%$; SP, 72.2\%; PLR, 2.4; NLR, 0.5; PPV, 67.4\%; NPV, 72.2\%) and that of patients with Gram-negative bacteremia was 0.720 (95\% CI, 0.624 to 0.803 ; $\mathrm{P}<0.001$; SS, 72.2\%; SP, $67.1 \%$; PLR, 2.2; NLR, 0.4; PPV, 53.1\%; NPV, 82.5\%). In both groups, cutoff values

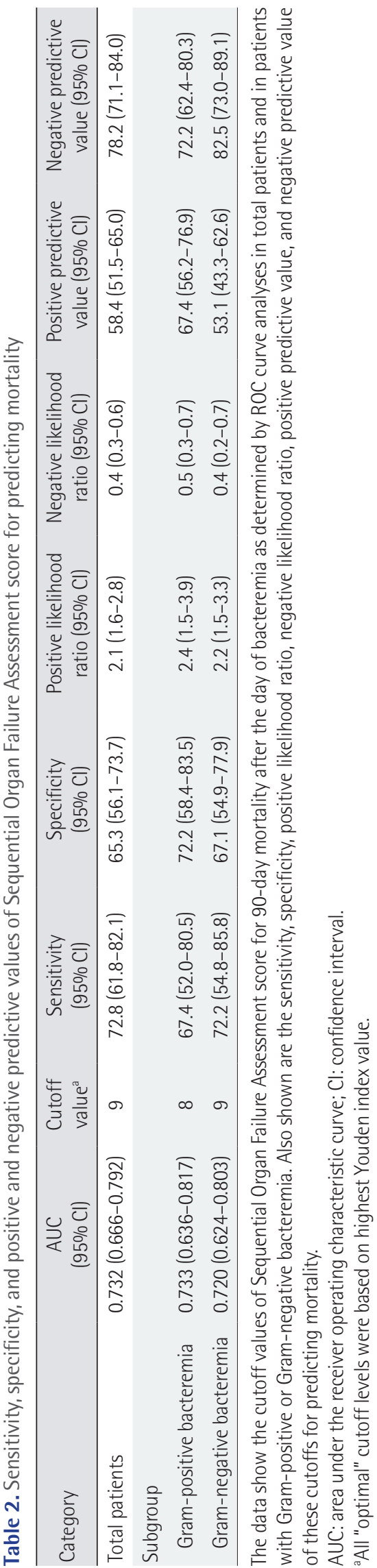

Acute and Critical Care 2020 August 35(3):169-178 
Table 3. Comparison of clinical characteristics between survivors and non-survivors after the day of bacteremia

\begin{tabular}{|c|c|c|c|}
\hline Characteristics & Survivor $(n=81)$ & Non-survivor $(n=121)$ & P-value \\
\hline \multicolumn{4}{|l|}{ Comorbidity $^{\mathrm{a}}$} \\
\hline Cardiovascular disease & $26(32.1)$ & $37(30.6)$ & 0.877 \\
\hline Diabetes mellitus & $25(30.9)$ & $30(24.8)$ & 0.420 \\
\hline Hemato-oncological diseases & $11(13.6)$ & $32(26.4)$ & 0.035 \\
\hline Cerebrovascular diseases & $13(16.0)$ & $18(14.9)$ & 0.844 \\
\hline Chronic renal diseases & $11(13.6)$ & $16(13.2)$ & $>0.999$ \\
\hline \multicolumn{4}{|l|}{ Main infection source ${ }^{a}$} \\
\hline Respiratory & $37(45.7)$ & $61(50.4)$ & 0.566 \\
\hline Vascular catheter-related & $27(33.3)$ & $19(15.7)$ & 0.006 \\
\hline Intra-abdominal & $3(3.7)$ & $22(18.2)$ & 0.002 \\
\hline Musculoskeletal and soft tissue & $7(8.6)$ & $8(6.6)$ & 0.595 \\
\hline Urinary & $5(6.2)$ & $4(3.3)$ & 0.489 \\
\hline \multicolumn{4}{|l|}{ Microorganism identified } \\
\hline Methicillin-resistant Staphylococcus aureus & $41(50.6)$ & $36(29.8)$ & 0.003 \\
\hline Vancomycin-resistant Enterococcus faecium & $5(6.2)$ & $20(16.5)$ & 0.030 \\
\hline Carbapenem-resistant Acinetobacter baumannii & $22(27.2)$ & $41(33.9)$ & 0.354 \\
\hline ESBL(+) Klebsiella pneumoniae & $9(11.1)$ & $16(13.2)$ & 0.828 \\
\hline ESBL(+) Escherichia coli & $7(8.6)$ & $11(9.1)$ & $>0.999$ \\
\hline Carbapenem-resistant Pseudomonas aeruginosa & 0 & $5(4.1)$ & 0.084 \\
\hline Requirement for vasopressors ${ }^{b}$ & $30(37.0)$ & $83(68.6)$ & $<0.001$ \\
\hline Requirement for hemodialysis $^{b}$ & $13(16.0)$ & $46(38.0)$ & 0.001 \\
\hline Requirement for neuromuscular blocking agents ${ }^{b}$ & $15(18.5)$ & $36(29.8)$ & 0.098 \\
\hline Consultation with an ID specialist regarding management of bacteremia & $61(75.3)$ & $67(55.4)$ & 0.005 \\
\hline $\operatorname{ARDS}^{b}$ & $47(58.0)$ & $106(87.6)$ & $<0.001$ \\
\hline
\end{tabular}

Values are presented as number (\%) and compared by chi-square tests.

ID: infectious diseases; ARDS: acute respiratory distress syndrome.

${ }^{a}$ Comparisons of the top five diseases; ${ }^{b}$ All clinical data were calculated or obtained from medical records on the day of bacteremia.

were 8 and 9, respectively (Table 2).

Comparisons of the clinical characteristics of survivors and non-survivors showed that the rates of hemato-oncologic diseases as comorbidities and intra-abdominal infections as main infectious sources were significantly higher in non-survivors. In addition, the rates of identification of Vancomycin-resistant Enterococcus faecium, requirements for vasopressors and hemodialysis on the day of bacteremia, and ARDS on the day of bacteremia were significantly higher in non-survivors (Table 3).

To evaluate the effect of SOFA score cutoff value on mortality, univariate and multivariate Cox regression models were constructed (Table 4). Multivariate Cox regression analyses of all patients and those with Gram-positive or Gram-negative bacteremia showed that SOFA score cutoff values were significantly associated with 90-day mortality rate (total patients: HR, 2.886 [95\% CI, 1.946 to 4.221; $\mathrm{P}<0.001]$, patients with Gram-pos- itive bacteremia: HR, 2.458 [95\% CI, 1.312 to 4.603; $\mathrm{P}<0.001$ ], patients with Gram-negative bacteremia: HR, 2.987 [95\% CI, 1.786 to 4.995$]$; $\mathrm{P}<0.001$ ). Kaplan-Meier analyses of 90-day survival yielded consistent results, showing the prognostic benefits of these cut-off values in all patients and those with Gram-positive or Gram-negative bacteremia (Figure 2).

\section{Comparison of SOFA Score with APACHE II Score on the Day of Bacteremia}

The present study also compared the prognostic utility of APACHE II score on the day of bacteremia with that of SOFA score for predicting mortality. For all patients, the AUC for APACHE II score was 0.662 (95\% CI, 0.593 to $0.727 ; \mathrm{P}<0.001$ ), which did not differ significantly from the AUC for SOFA score. Subgroup analyses, however, showed that the AUC for predicting mortality was significantly higher for SOFA score than for APACHE 
Table 4. Univariate and multivariate analysis of factors associated with mortality in all patients and in patients with Gram-positive or Gram-negative bacteremia

\begin{tabular}{|c|c|c|c|c|}
\hline \multirow{2}{*}{ Factor } & \multicolumn{2}{|c|}{ Univariate analysis } & \multicolumn{2}{|c|}{ Multivariate analysis } \\
\hline & HR (95\% Cl) & P-value & HR (95\% Cl) & P-value \\
\hline \multicolumn{5}{|l|}{ Total patients } \\
\hline SOFA score $\geq 9^{a}$ & $3.099(2.123-4.523)$ & $<0.001$ & $2.886(1.946-4.221)$ & $<0.001$ \\
\hline No infectious disease consultation & $2.193(1.528-3.146)$ & $<0.001$ & $2.261(1.534-3.331)$ & $<0.001$ \\
\hline Intra-abdominal infections as source of infection & $2.614(1.637-4.172)$ & $<0.001$ & $2.023(1.252-3.268)$ & 0.004 \\
\hline Requirement for NMBAs ${ }^{a}$ & $1.629(1.102-2.409)$ & 0.014 & & \\
\hline Hemato-oncologic diseases as comorbidities & $1.714(1.142-2.572)$ & 0.009 & & \\
\hline \multicolumn{5}{|l|}{ Gram-positive bacteremia } \\
\hline SOFA score $\geq 8^{\mathrm{a}}$ & $3.206(1.763-5.831)$ & $<0.001$ & $2.458(1.312-4.603)$ & 0.005 \\
\hline Age $\geq 65$ years & $2.251(1.311-3.866)$ & 0.003 & $1.942(1.116-3.379)$ & 0.019 \\
\hline Hemato-oncologic malignancies as comorbidities & $4.032(2.059-7.894)$ & $<0.001$ & & \\
\hline Requirement for NMBAs ${ }^{a}$ & $1.875(1.053-3.341)$ & 0.033 & & \\
\hline Intra-abdominal infections as source of infection & $5.473(2.390-12.535)$ & $<0.001$ & & \\
\hline No infectious disease consultation & $1.767(1.025-3.046)$ & 0.040 & & \\
\hline \multicolumn{5}{|l|}{ Gram-negative bacteremia } \\
\hline SOFA score $\geq 9^{a}$ & $3.129(1.886-5.189)$ & $<0.001$ & $2.987(1.786-4.995)$ & $<0.001$ \\
\hline No infectious disease consultation & $2.709(1.677-4.376)$ & $<0.001$ & $2.671(1.641-4.348)$ & $<0.001$ \\
\hline Intra-abdominal infections as source of infection & $1.785(1.005-3.171)$ & 0.048 & & \\
\hline
\end{tabular}

Statistical significance was determined by univariate and multivariate Cox proportional hazards models. Mortality was defined as mortality 90 days after the day of blood culture.

HR: hazard ratio; Cl: confidence interval; SOFA: Sequential Organ Failure Assessment; NMBA: neuromuscular blocking agent.

${ }^{a}$ All clinical data were calculated or obtained from medical records on the day of bacteremia.

II score in patients with Gram-negative, but not Gram-positive, bacteremia (Figure 3).

\section{DISCUSSION}

The present study found that SOFA score on the day of bacteremia can predict the risk of mortality in ventilated patients with MDR bacteremia. Based on the ROC curve, this score may be an appropriate predictor of 90-day mortality after bacteremia. To our knowledge, this is the first comprehensive study to evaluate the clinical ability of SOFA score to predict long-term mortality in ventilated patients with MDR bacteremia in our country.

Also, this study found that the SOFA score cutoff values for predicting 90-day mortality differed in the total patient population and in the subgroups with Gram-positive or Gram-negative bacteremia. Although multivariate Cox regression analyses showed different prognostic factors in these subgroups, SOFA score cut-offs were significant prognostic indicators in both subgroups. In addition, Kaplan-Meier analysis showed that 90-day mortality rates were about 2-fold higher in patients with SOFA scores above than below these cut-offs. Taken together, these findings suggest that SOFA score on the day of bacteremia may help in future care planning for these patients.

There is literature regarding the SOFA score cutoff values for predicting mortality in patients with Gram-negative bacteremia. Chen et al. [19] evaluated patients with carbapenemresistant Acinetobacter baumannii bacteremia, and calculated the effectiveness of SOFA score at the onset of bacteremia in predicting the mortality. They reported that ventilator care was performed in $63.8 \%$ patients and SOFA scores $>8$ and $>7$ were associated with significantly higher 14-day and in-hospital mortality rates, respectively [19]. Their study design and the SOFA score cutoff levels were similar to the present study. However, the enrollment criteria were different, and the SOFA cutoff value was evaluated for predicting 90-day mortality in our study.

Our study also found that ROC curves of the SOFA score were significantly higher than those of the APACHE II score for predicting 90-day mortality in patients with Gram-negative bacteremia, which was consistent with a previous report [19]. Our data suggest that SOFA score would be more useful 

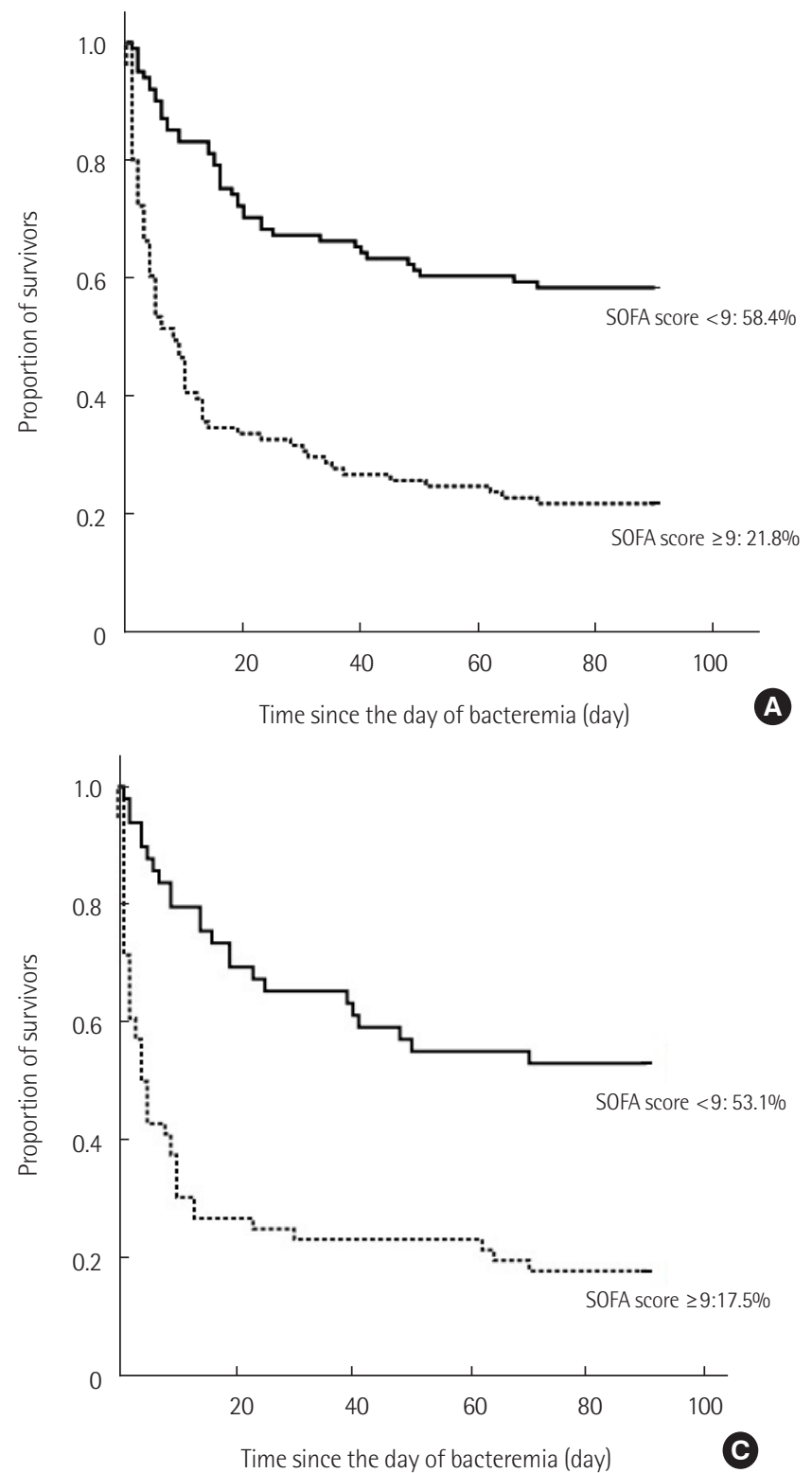

for predicting mortality in ventilated patients with Gram-negative bacteremia. However, the present study was a retrospective design, and large-scale, multicenter investigations are needed to determine whether the SOFA score would be a more useful prognostic indicator than other severity-of-illness scoring systems in MDR bacteremia patients.

In addition to SOFA score, we sought to select the best subset of prognostic indicators, including baseline demographic factors, comorbidities, and clinical parameters on the day of bacteremia, and to develop models predicting short-term and/or long-term mortality. None of these factors differed significantly, however, which again may have been due to the small number of patients. SOFA score is not ideal for assessing prognosis because it does not take pre-existing comorbidities

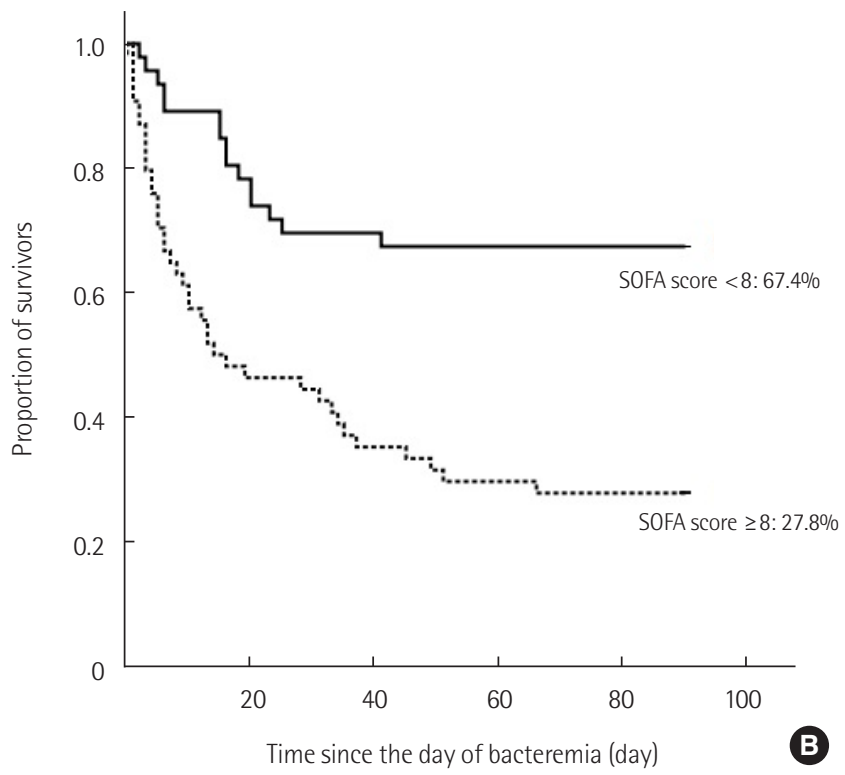

Figure 2. Kaplan-Meier analysis of 90-day survival after the day of bacteremia according to Sequential Organ Failure Assessment (SOFA) score cut-off values in (A) all enrolled patients, (B) Grampositive patients, and (C) Gram-negative patients. Differences between patients above and below the cut-offs were (A) 39.39\% $(\mathrm{P}<0.001)$, (B) 16.53\% $(\mathrm{P}<0.001)$, and $(\mathrm{C}) 22.69 \%(\mathrm{P}<0.001)$ by log-rank tests.

into account. Rather, a new, expanded prognostic model, including comorbidities, sources of infection, and various clinical parameters, will be necessary to increase the ability to predict short- and long-term mortality.

The present study showed that roughly $60 \%$ of enrolled patients consulted an infectious diseases specialist for selection of optimal antibiotics. Although the percentages of patients with Gram-positive and Gram-negative bacteremia who consulted a specialist did not differ significantly (66.0\% vs. $61.9 \%$, $\mathrm{P}=0.664$ ), consultation was especially associated with a reduced mortality rate in patients with Gram-negative bacteremia. Although several previous studies have reported that consultation was associated with reduced in-hospital mortality rates and overall hospital costs $[20,21]$, few antimicrobial 


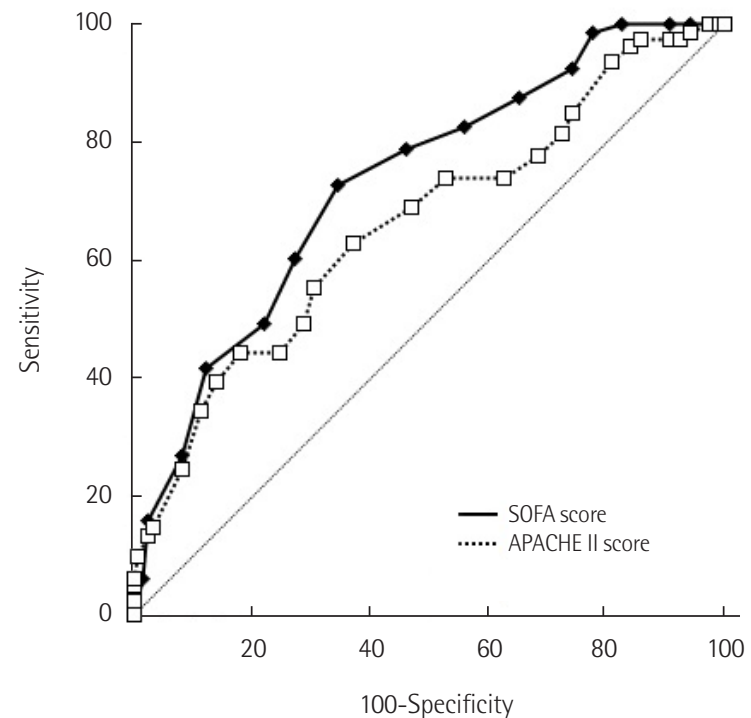

A

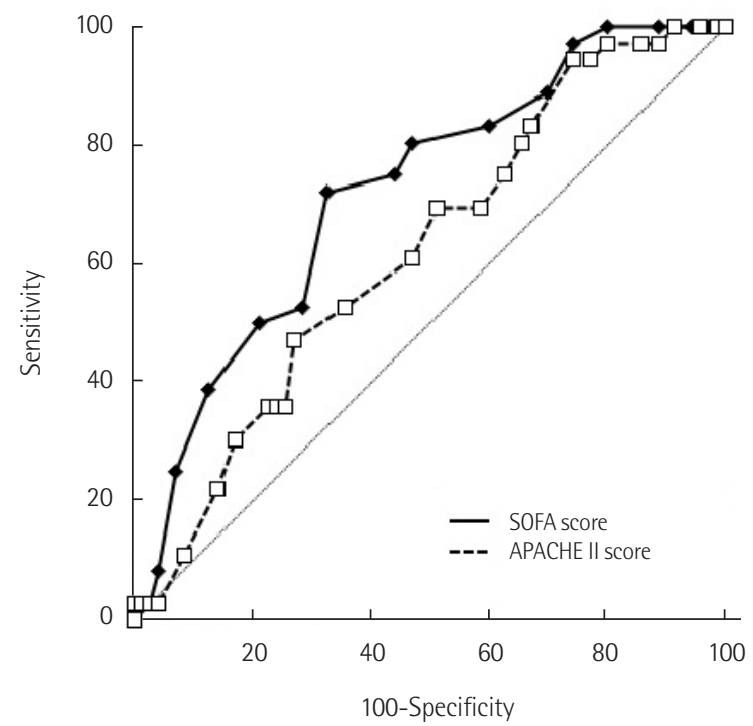

C

stewardship programs have been established at university-affiliated hospitals in Korea, despite their increasing consumption of empirical broad-spectrum antibiotics. The present findings suggest that antibiotic stewardship programs are needed to control the increasing prevalence of MDR bacteria in our country.

This study had several limitations. First, the study was single-centered and retrospective. In addition, SOFA scores were determined at a university-affiliated tertiary care. Therefore, it may not be possible to generalize our data to other settings, although most Korean patients with bacteremia are managed in tertiary care hospitals. Second, careful interpretation is necessary in clinical meanings of the SOFA score because total enrolled patients were from six functionally separate ICUs with

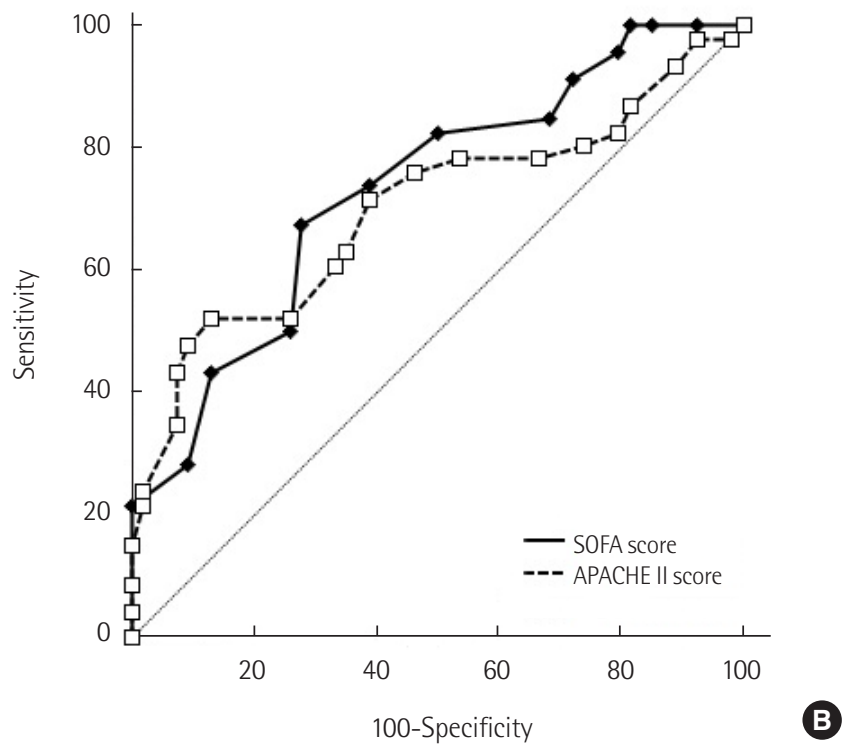

Figure 3. Receiver operating characteristic (ROC) curves for Sequential Organ Failure Assessment (SOFA) and Acute Physiology and Chronic Health Evaluation (APACHE) II scores for predicting 90-day mortality after the day of bacteremia in (A) all enrolled patients, (B) Gram-positive patients, and (C) Gram-negative patients. (A) In all patients, the area under the ROC curves for the SOFA and APACHE II scores were 0.732 (95\% confidence interval [Cl], 0.666-0.792; $\mathrm{P}<0.001)$ and 0.662 (95\% Cl, 0.593-0.727; $\mathrm{P}<$ $0.001)$, respectively, with no difference between the two $(P=0.059)$. (B) In patients with Gram-positive bacteremia, the AUCs for the SOFA and APACHE II scores were 0.733 (95\% Cl, 0.636-0.817; $\mathrm{P}<0.001)$ and $0.701(95 \% \mathrm{Cl}, 0.601-0.788 ; \mathrm{P}<0.001)$, respectively, with no difference between the two $(P=0.572)$. (C) In patients with Gram-negative bacteremia, the AUCs for the SOFA and APACHE II scores were $0.720(95 \% \mathrm{Cl}, 0.624-0.803 ; \mathrm{P}<0.001)$ and 0.622 (95\% Cl, 0.523-0.714; $\mathrm{P}<0.001)$, respectively. The AUC for SOFA score was significantly higher than the AUC for APACHE II score $(P=0.037)$.

different patient subgroups. Third, this study was a retrospective design, and we could not accurately measure the SOFA score, especially the Glasgow Coma Scale or $\mathrm{PaO}_{2} / \mathrm{FiO}_{2}$ ratio, which may result in a selection bias. Fourth, other clinical factors (in addition to the cutoff values of SOFA score) were associated with prognosis as shown in Table 4 . These factors were different in patients with Gram-positive and Gram-negative bacteremia, respectively. We could not accurately analyze these differences in prognostic factors in both groups due to the retrospective design. Fifth, we hypothesized that the SOFA score would be a more useful prognostic factor in some subgroups, including patients with ARDS, those aged $\geq 65$ years, and those with hemato-oncologic malignancies as comorbidities. However, none of these factors differed significantly in 
survivors and non-survivors, a finding that may be attributed to the small number of enrolled patients.

In conclusion, SOFA score on the day of bacteremia may be a useful prognostic indicator for predicting 90-day mortality in ventilated patients with MDR bacteremia. Large-scale multicenter studies are needed to determine whether SOFA score is a prognostic indicator of short-term and/or long-term mortality as well as to compare SOFA score with other scoring methods for predicting prognosis.

\section{CONFLICT OF INTEREST}

No potential conflict of interest relevant to this article was reported.

\section{ACKNOWLEDGMENTS}

This work was supported by the National Research Foundation of Korea (NRF) grant funded by the Korean government (Ministry of Science and ICT) (2016R1C1B1008529).

\section{ORCID}

Yeseul Oh https://orcid.org/0000-0002-9777-3258

Jiyeon Roh https://orcid.org/0000-0002-1595-9535 Jaemin Lee https://orcid.org/0000-0003-1022-0667 Hyun Sung Chung https://orcid.org/0000-0002-8834-7302 Kwangha Lee https://orcid.org/0000-0001-9878-201X Min Ki Lee https://orcid.org/0000-0002-0163-115X

\section{AUTHOR CONTRIBUTIONS}

Conceptualization: YO, KL. Data curation: YO, JR, JL, HSC. Formal analysis: YO, KL. Funding acquisition: KL, MKL. Methodology: YO, JR, JL, HSC. Project administration: KL, MKL. Visualization: YO, KL. Writing-original draft: YO, JR, JL, HSC. Writing-review \& editing: YO, KL.

\section{REFERENCES}

1. Pulcini C, Defres S, Aggarwal I, Nathwani D, Davey P. Design of a 'day 3 bundle' to improve the reassessment of inpatient empirical antibiotic prescriptions. J Antimicrob Chemother 2008;61:1384-8.

2. Levy-Hara G, Amábile-Cuevas CF, Gould I, Hutchinson J, Abbo L, Saxynger L, et al. "Ten commandments" for the appropriate use of antibiotics by the practicing physician in an outpa- tient setting. Front Microbiol 2011;2:230.

3. Levy Hara G, Kanj SS, Pagani L, Abbo L, Endimiani A, Wertheim HF, et al. Ten key points for the appropriate use of antibiotics in hospitalised patients: a consensus from the Antimicrobial Stewardship and Resistance Working Groups of the International Society of Chemotherapy. Int J Antimicrob Agents 2016;48:239-46.

4. Magill SS, Edwards JR, Bamberg W, Beldavs ZG, Dumyati G, Kainer MA, et al. Multistate point-prevalence survey of health care-associated infections. N Engl J Med 2014;370:1198-208.

5. Magiorakos AP, Srinivasan A, Carey RB, Carmeli Y, Falagas ME, Giske CG, et al. Multidrug-resistant, extensively drug-resistant and pandrug-resistant bacteria: an international expert proposal for interim standard definitions for acquired resistance. Clin Microbiol Infect 2012;18:268-81.

6. Brusselaers N, Vogelaers D, Blot S. The rising problem of antimicrobial resistance in the intensive care unit. Ann Intensive Care 2011;1:47.

7. Kim JH, Hong SK, Kim KC, Lee MG, Lee KM, Jung SS, et al. Influence of full-time intensivist and the nurse-to-patient ratio on the implementation of severe sepsis bundles in Korean intensive care units. J Crit Care 2012;27:414.e11-21.

8. Kwak SH, Jeong CW, Lee SH, Lee HJ, Koh Y. Current status of intensive care units registered as critical care subspecialty training hospitals in Korea. J Korean Med Sci 2014;29:431-7.

9. Lim CM, Kwak SH, Suh GY, Koh Y. Critical care in Korea: present and future. J Korean Med Sci 2015;30:1540-4.

10. Vincent JL, Moreno R, Takala J, Willatts S, De Mendonca A, Bruining $\mathrm{H}$, et al. The SOFA (Sepsis-related Organ Failure Assessment) score to describe organ dysfunction/failure. On behalf of the Working Group on Sepsis-Related Problems of the European Society of Intensive Care Medicine. Intensive Care Med 1996;22:707-10.

11. Singer M, Deutschman CS, Seymour CW, Shankar-Hari M, Annane D, Bauer M, et al. The third international consensus definitions for sepsis and septic shock (Sepsis-3). JAMA 2016; 315:801-10.

12. Knaus WA, Draper EA, Wagner DP, Zimmerman JE. APACHE II: a severity of disease classification system. Crit Care Med 1985;13:818-29.

13. Acute Respiratory Distress Syndrome Network, Brower RG, Matthay MA, Morris A, Schoenfeld D, Thompson BT, et al. Ventilation with lower tidal volumes as compared with traditional tidal volumes for acute lung injury and the acute respiratory distress syndrome. N Engl J Med 2000;342:1301-8.

14. Lockwood AM, Perez KK, Musick WL, Ikwuagwu JO, Attia E, Fasoranti OO, et al. Integrating rapid diagnostics and antimi- 
crobial stewardship in two community hospitals improved process measures and antibiotic adjustment time. Infect Control Hosp Epidemiol 2016;37:425-32.

15. ARDS Definition Task Force, Ranieri VM, Rubenfeld GD, Thompson BT, Ferguson ND, Caldwell E, et al. Acute respiratory distress syndrome: the Berlin Definition. JAMA 2012;307:252633.

16. Howard P, Pulcini C, Levy Hara G, West RM, Gould IM, Harbarth S, et al. An international cross-sectional survey of antimicrobial stewardship programmes in hospitals. J Antimicrob Chemother 2015;70:1245-55.

17. Perkins NJ, Schisterman EF. The inconsistency of "optimal" cutpoints obtained using two criteria based on the receiver operating characteristic curve. Am J Epidemiol 2006;163:670-5.

18. DeLong ER, DeLong DM, Clarke-Pearson DL. Comparing the areas under two or more correlated receiver operating char- acteristic curves: a nonparametric approach. Biometrics 1988; 44:837-45.

19. Chen SJ, Chao TF, Chiang MC, Kuo SC, Chen LY, Yin TL, et al. Prediction of patient outcome from Acinetobacter baumannii bacteremia with Sequential Organ Failure Assessment (SOFA) and Acute Physiology and Chronic Health Evaluation (APACHE) II scores. Intern Med 2011;50:871-7.

20. Saunderson RB, Gouliouris T, Nickerson EK, Cartwright EJ, Kidney A, Aliyu SH, et al. Impact of routine bedside infectious disease consultation on clinical management and outcome of Staphylococcus aureus bacteraemia in adults. Clin Microbiol Infect 2015;21:779-85.

21. Fries BL, Licitra C, Crespo A, Akhter K, Busowski MT, Salazar $\mathrm{D}$, et al. Infectious diseases consultation and the management of Staphylococcus aureus bacteremia. Clin Infect Dis 2014;58: 598-9. 